\title{
Comparison of Nitrogen Bioaccessibility from Salmon and Whey Protein Hydrolysates using a Human Gastrointestinal Model (TIM-1)
}

\author{
Bomi Framroze, ${ }^{1} *$ Patricia Savard, ${ }^{2}$ Diane Gagnon, ${ }^{2}$ \\ Véronique Richard, ${ }^{2}$ and Sylvie F. Gauthier ${ }^{2}$ \\ ${ }^{1}$ Hofseth Biocare AS, Molovegen 6, 6000 Ålesund, Norway; ${ }^{2}$ Institute of Nutrition and \\ Functional Foods (INAF), 2440 Hochelaga Blvd., Université Laval, Quebec city, QC, Canada \\ G1V 0A6
}

*Corresponding author: Dr. Bomi Framroze, CSO, Hofseth Biocare AS, Molovegen 6, 6000 Ålesund, Norway

Submission date: April 13, 2014; Acceptance date: May 23, 2014; Publication date: May 26, 2014

\begin{abstract}
Background: The TIM-1 system is a computer-controlled multi-compartmental dynamic model that closely simulates in vivo gastrointestinal tract digestion in humans. During digestion, the compounds released from meal matrix by gastric and intestinal secretions (enzymes) are progressively absorbed through semipermeable membranes depending on their molecular weight. These absorbed (dialysed) compounds are considered as bioaccessible, which means that they can be theoretically absorbed by the small intestine in the body.
\end{abstract}

Methods: Salmon protein hydrolysate (SPH), whey protein hydrolysates extensively (WPHHigh) or weakly (WPH-Low) hydrolysed, non-hydrolysed whey protein isolate (WPI) and mixtures of WPI:SPH (90:10, 80:20) were digested in TIM-1 using the conditions for a fast gastrointestinal transit that simulate the digestion of a liquid meal in human adults. During digestion ( 2 hours), samples were collected in intestinal compartments (duodenum, jejunum, and ileum) and in both jejunal and ileal dialysates to determine their nitrogen content. All the products were compared in terms of kinetics of nitrogen absorption through the semipermeable membranes (bioaccessible nitrogen) and nitrogen distribution throughout the intestinal compartments at the end of the 2 hour digestion.

Results: After a 2 h-digestion in TIM-1, SPH was the protein substrate from which the highest amount of nitrogen $(67.0 \%)$ becomes available for the small intestine absorption. WPH-High had the second highest amount $(56.0 \%)$ of bioaccessible nitrogen while this amount decreased to $38.5-42.2 \%$ for the other protein substrates. The high nitrogen bioaccessibility of SPH is consistent with its richness in low molecular weight peptides $(50 \%<1000 \mathrm{Da})$.

Conclusions: The results of this study indicate that SPH provides a higher proportion of bioaccessible nitrogen to a healthy adult compared to all forms of whey proteins, including extensively hydrolysed whey protein hydrolysate. The substitution of non-hydrolysed WPI by 
small amounts of SPH (10-20\%) improved slightly its nitrogen bioaccessibility, making the mixture particularly suitable for applications such as medical foods that require rapid protein uptake and where the use of extensively hydrolysed whey protein is unfeasible due to its undesirable organoleptic properties.

Keywords: Salmon protein hydrolysate, Whey protein, Nitrogen bioaccessibility, TIM-1

\section{BACKGROUND:}

TIM-1 is a computer-controlled multi-compartmental dynamic model that closely simulates the conditions of the in vivo gastrointestinal tract in humans and monogastric animals [1]. It has been developed at the TNO Nutrition \& Food Research Institute (Zeist, The Netherlands). This gastrointestinal (GI) model reproduces the physiological conditions of digestion such as meal size, peristaltic movements, $\mathrm{pH}$, composition of the gastric and intestinal secretions, gastrointestinal transit, and small intestine absorption of water and digested products by their passage through semipermeable membranes, which are connected to the jejunal and ileal compartments. With this in vitro GI model, digested products measured in the jejunal and ileal dialysates can be considered as bioaccessible, which means that they can be theoretically assimilated by the body. In fact, "bioaccessibility" was previously defined as the proportion of a compound that is released from a food matrix in the gastrointestinal tract and thus becomes available for intestinal absorption and that can further enter the blood stream [2].

The different components of the TIM-1 have been variously described and many illustrative schematics have been published [3-5]. This model has been utilized in several digestion studies to evaluate the digestibility/availability/absorption of minerals $[6,7]$, vitamins [8, 9], proteins [10-12], and milk growth factors [13]. TIM-1 has also been used to investigate nutraceuticals [14-16] and drug delivery $[5,17,18]$.

\section{INTRODUCTION:}

The use of salmon offcuts from the salmon fillet industry to produce human grade nutritional products is an aspirational goal of sustainability for the Norwegian salmon aquaculture industry. Head and backbone salmon offcuts, produced after filleting, were subjected to enzymatic hydrolysis followed by separation of the water-soluble protein phase and spray-drying the concentrate to produce a very palatable salmon protein hydrolysate powder (SPH). This hydrolysate contained oligopeptides and peptides with a molecular weight of less than 3000 Daltons, as determined by MALDI-TOF (Matrix Assisted Laser Desorption/Ionization-Time Of Flight) analysis. Gel permeation chromatography analysis further showed that $50 \%$ of these peptides have a molecular weight of less than 1000 Daltons [19].

Whey protein hydrolysates (WPH) are known for their bioactivity and functionality [20] but their bitter taste, particularly when they are extensively hydrolysed, represents a major limit to their utilisation as ingredients in food and nutritional formulations. In fact, bitterness in a WPH was recently associated with four main peptides that were reported to explain $88 \%$ of the bitterness' product intensity [21]. Since bitter peptides are included in the sequence of whey proteins and released by the action of proteases during the manufacture of WPH, it is extremely 
difficult to prevent the development of bitterness in the final product. Different approaches were tested to reduce the bitterness of WPH, such as the addition of bitter taste inhibiting agents in WPH beverages, but the effectiveness of these inhibitors varied widely depending on the extent of hydrolysis of WPH and the components present in the final beverages [22]. Actually, WPH bitterness thus remains the main limiting factor for their use in food and pharmaceutical applications.

In the present study, nitrogen bioaccessibility from different protein substrates was determined using TIM-1 under conditions simulating the ingestion of a liquid meal (fast transit). The SPH was compared to a non-hydrolysed whey protein isolate (WPI) and to WPI hydrolysates extensively (WPH-High) and weakly (WPH-Low) hydrolysed. Two substitution levels (10\% and 20\%) of WPI by SPH were tested in order to verify the nitrogen bioaccessibility when SPH is provided in a mixed protein powder.

The hypothesis is that nitrogen bioaccessibility from SPH will be higher than WPH-High during its in vitro gastrointestinal digestion and that a small addition of SPH (10-20\%) in a nonhydrolysed WPI should synergistically increase the nitrogen bioaccessibility in comparison with WPI digested alone.

\section{MATERIALS AND METHODS:}

Materials: The enzymes used for the digestion of proteins were pepsin from porcine gastric mucosa (EC 3.4.23.1; 3,200-4,500 U/mg protein, Sigma-Aldrich, MO, USA), trypsin from porcine pancreas (EC 3.4.21.4; $\geq 7,500 \mathrm{U} / \mathrm{mg}$ protein, Sigma-Aldrich), and partially purified porcine pancreatin (Pancrex V Powder, Pains and Byrne, Greenford, England). According to the manufacturer, 1 gram of Pancrex V contains 1,400, 25,000 and 30,000 British Pharmacopeia units of free protease, lipase, and amylase, respectively.

The proteins used in the study were: a salmon protein hydrolysate (SHP, lot 1221, Hofseth Biocare SA, Aalesund, Norway) containing 90.0\% protein (normalized); an instant whey protein isolate 90 (WPI, lot FL1124, Milk Specialities Global, MN, USA) containing 85.7\% protein (normalized); a whey protein hydrolysate extensively hydrolysed (WPH-High, lot 1568641, Lacprodan DI-3095, Arla Foods Ingredients, Viby J, Denmark) containing 83.5\% protein (normalized); a whey protein hydrolysate weakly hydrolysed (WPH-Low, lot U480214, Lacprodan DI-6171, Arla Foods Ingredients) containing 86.1\% protein (normalized).

Description of the TIM-1 Model: TIM-1 includes four compartments corresponding to the stomach, duodenum, jejunum and ileum, which are connected in series. Each compartment comprises a flexible wall within a glass jacket to allow an efficient heat transfer. During digestion, meal progresses through the compartments and is subjected to peristaltic-like movement by means of water pressure on the outside of the flexible wall. The computercontrolled parameters during digestion were: temperature $\left(37^{\circ} \mathrm{C}\right), \mathrm{pH}$ values, secretion of gastric and intestinal electrolytes containing enzymes, peristaltic cycles, and the flow rate of the chyme throughout the system. The jejunal and ileal compartments were connected to hollow-fibre membranes (Baxter Xenium 110 high-flux dialyzer, Baxter Healthcare, Deerfield, US) with a molecular weight cut-off (MWCO) of $11.8 \mathrm{kDa}$. These semipermeable membranes simulate the absorption of water and digestion products from the chyme and modify its electrolyte 
concentrations. Nitrogen present in jejunal and ileal dialysates constituted the fraction of bioaccessible nitrogen. After the passage of the chyme throughout the system, the effluent residue, representing the portion of meal that reach colon, is discharged in a bottle kept on ice.

Digestion of Protein Substrates: Protein substrate $(25 \mathrm{~g})$ was solubilized in reverse osmosis water $(300 \mathrm{~g})$ under agitation for 5 minutes. An aliquot $(5 \mathrm{~g})$ of this solution was kept to determine nitrogen content in the starting meal, while the rest of the meal $(320 \mathrm{~g})$ was fed into the TIM-1 gastric compartment. When mixtures of WPI and SPH were digested, the protein substrates were combined according to ratios of 90:10 (22.5 g WPI + 2.5 g SPH) and 80:20 (20 g $\mathrm{WPI}+5 \mathrm{~g} \mathrm{SPH})$ before their solubilisation in the same amount of water $(300 \mathrm{~g})$.

During digestion, gastric and duodenal secretions were delivered to the respective compartments at a flow rate of $0.25 \mathrm{ml} / \mathrm{min}$. Gastric secretions consisted of pepsin $(0.28 \mathrm{mg} / \mathrm{ml})$ in an electrolytes solution ( $\left.\mathrm{NaCl}, 3.0 \mathrm{~g} / \mathrm{l} ; \mathrm{KCl}, 1.1 \mathrm{~g} / \mathrm{l} ; \mathrm{CaCl}_{2}, 0.15 \mathrm{~g} / \mathrm{l} ; \mathrm{NaHCO}_{3}, 0.60 \mathrm{~g} / \mathrm{l}\right)$ while duodenal secretions consisting of $7 \%$ pancreatin solution (Pancrex $\mathrm{V}$ powder) in intestinal electrolytes solution $\left(\mathrm{NaCl}, 5.0 \mathrm{~g} / \mathrm{l} ; \mathrm{KCl}, 0.60 \mathrm{~g} / \mathrm{l} ; \mathrm{CaCl}_{2}, 0.30 \mathrm{~g} / \mathrm{l} ; \mathrm{pH} 7.0\right)$. Both electrolytes solutions were prepared in reverse osmosis-purified water. Meal digestion lasted $2 \mathrm{~h}$ and digested samples were collected in the intestinal compartments for their nitrogen content analysis as shown in Table 1. Chyme remaining in duodenal, jejunal and ileal compartments at the end of the digestion (120 min) was sampled for subsequent nitrogen analysis, while samples were collected at different times in the dialysates (jejunal and ileal) in order to measure the kinetics of nitrogen absorption through the semipermeable membranes. For the effluent residue, which contains the non-bioaccessible nitrogen, the totality of the fraction ( 0 to $120 \mathrm{~min}$ ) collected during the two hour digestion was analysed for its nitrogen content.

Table 1. Time intervals for sampling in intestinal compartments.

\begin{tabular}{ll}
\hline Compartment & $\begin{array}{c}\text { Sampling times } \\
\text { (min) }\end{array}$ \\
\hline Duodenum (chyme) & 120 \\
Jejunum (chyme) & 120 \\
Ileum (chyme) & 120 \\
Jejunal membrane (dialysate) & $0,30,60,90,120$ \\
Ileal membrane (dialysate) & $0,30,60,90,120$ \\
Effluent (residue) & 0 to 120 \\
\hline
\end{tabular}

All the samples collected during digestion were frozen at $-80^{\circ} \mathrm{C}$ until their nitrogen analysis. A blank digestion run was performed using the same conditions but by adding $300 \mathrm{~g}$ of reverse osmosis water in the stomach compartment. This blank allows the estimation of nitrogen content in digestive secretions (e.g. enzymes) for the calculation of nitrogen distribution throughout the TIM-1 compartments during the digestion. All the protein substrates and mixtures of WPI:SPH 
were digested in duplicate. Meals were digested using the conditions for a fast gastrointestinal transit as shown in Table 2.

Table 2. TIM-1 conditions for a fast gastrointestinal transit simulating the digestion of a liquid meal in adults.

\begin{tabular}{ll}
\hline Gastric compartment: & \\
\hline Time/pH & $0 \mathrm{~min} / 4.5$ \\
& $10 \mathrm{~min} / 3.2$ \\
& $20 \mathrm{~min} / 2.8$ \\
& $40 \mathrm{~min} / 1.8$ \\
& $60 \mathrm{~min} / 1.7$ \\
& $120 \mathrm{~min} / 1.5$ \\
& $30 \mathrm{~min}$ \\
$\mathrm{t}_{1 / 2}$ (delivery half-time) & 1 \\
\hline Intestinal compartments: & \\
\hline Compartment/pH & Duodenum/6.5 \\
& Jejunum/6.8 \\
& Ileum/7.2 \\
$\mathrm{t}_{1 / 2}$ (delivery half-time) & $150 \mathrm{~min}$ \\
\hline
\end{tabular}

Nitrogen analysis: Liquid samples were thawed then aliquots $(1 \mathrm{ml})$ were transferred in microtubes for their drying during 5 hours in a Speedvac Concentrator (Savant SPD13DDA, Thermo Scientific, Asheville, NC, USA). Dry samples were rehydrated in $0.5 \mathrm{ml}$ of water and their nitrogen content determined by the Dumas combustion method [23] using a Truspec nitrogen analyzer (Leco Corp., MI, USA).

Calculations: Nitrogen (N) content of the samples collected in the different TIM-1 compartments during the $2 \mathrm{~h}$-digestion, expressed as percentage (\%) of nitrogen contained in the starting meal, was determined using the following equation:

Nitrogen content $\left(\%\right.$ of $\left.\mathrm{N}_{\text {meal }}\right)=\frac{\left(\mathrm{N}_{\text {sample }}-\mathrm{N}_{\text {blank }}\right)}{\mathrm{N}_{\text {meal }}} \times 100$

\section{RESULTS AND DISCUSSION:}

The results for bioaccessible nitrogen were compared on the basis of only the nitrogen absorbed through the semipermeable membranes connected to jejunal and ileal compartments. Since the rate of nitrogen absorption through these membranes can vary during digestion but also depends on the type of protein substrate, nitrogen content in intestinal dialysates (jejunal + ileal) was measured at different times during the two hour digestion (Figure 1). 


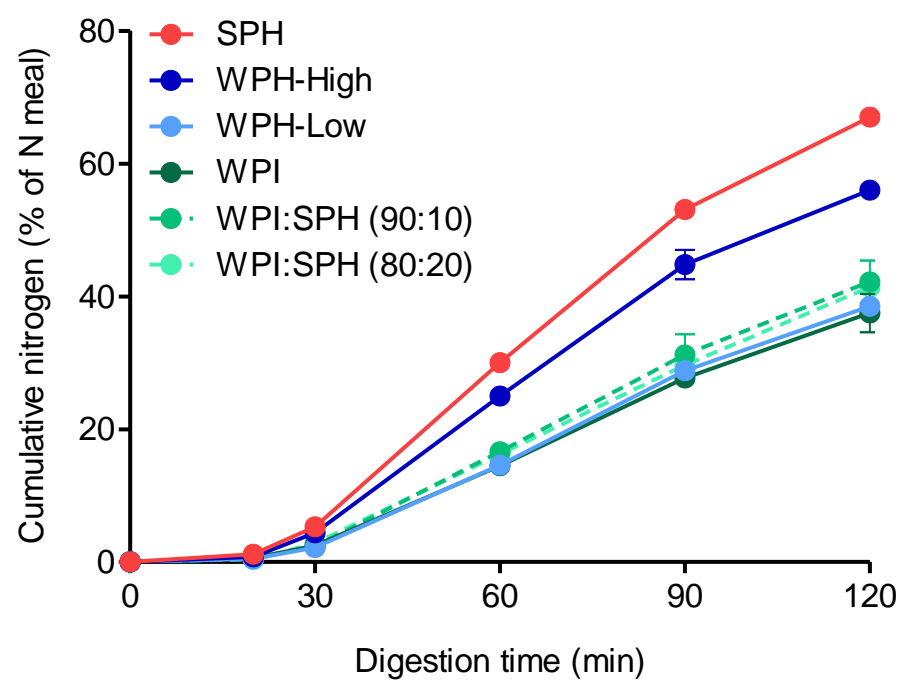

Figure 1. Cumulative nitrogen content in jejunal + ileal dialysates measured at different times during the $2 \mathrm{~h}$-digestion. SHP, salmon protein hydrolysate; WPH-High, whey protein hydrolysate extensively hydrolysed; WPH-Low, whey protein hydrolysate weakly hydrolysed, WPI, whey protein isolate. Values are means \pm standard deviation ( $n=2$ digestions).

Figure 1 shows that the cumulative nitrogen content in jejunal + ileal dialysates increased progressively during the digestion. For all the protein substrates, but in particular for SPH and WPI-High, a decreased in the rate of nitrogen absorption was observed after 90 minutes, which can be explained by the low amount of nitrogen remaining in the chyme that disadvantages the dialysis process through the semipermeable membranes. At the end of the two hour digestion, SPH was the protein substrate from which the highest amount of nitrogen $(67.0 \%)$ became bioaccessible. Whey protein hydrolysate extensively hydrolysed (WPH-High) gave intermediate values with $56.0 \%$ of bioaccessible nitrogen while the other substrates (WPI; WPH-Low; 90:10 and 80:20 mixtures of WPI:SPH) gave lower but similar amounts of bioaccessible nitrogen with values varying from $37.5 \%$ to $42.2 \%$. As expected, the lowest amount of bioaccessible nitrogen was measured for non-hydrolysed WPI (37.5\%). Surprisingly, the amount of bioaccessible nitrogen in weakly hydrolysed WPH $(38.5 \%)$ was similar to that of non-hydrolysed WPI $(37.5 \%)$, indicating that whey proteins in this product are probably a mixture of intact molecules and large molecular weight peptides. According to the manufacturer's specifications, the degree of hydrolysis of this product is $4.3 \%$. The substitution of non-hydrolysed WPI by small proportion (10\% and 20\%) of SPH increased slightly the amount of bioaccessible nitrogen from $\sim 42.0 \%$ in the mixtures to $37.5 \%$ in WPI. Finally, it must be emphasized that low standard deviations of the values were obtained, indicating that TIM-1 is a very reproducible model.

Figure 2 enables a comparison of the nitrogen distribution (\%) in the intestinal compartments of the TIM-1 at the end of the digestion ( 2 hours) for the different protein substrates. This figure clearly shows that SPH released the highest amount (67.0\%) of bioaccessible nitrogen (jejunal + ileal dialysates) after the two hour digestion compared to the others protein substrates $(<56.0 \%)$. For all the substrates, the proportion of bioaccessible nitrogen was higher in jejunal dialysate than in ileal dialysate, indicating that the proteins are 
rapidly digested by the gastric and intestinal enzymes to release low molecular weight peptides that can be absorbed by the permeable membrane connected to the jejunum compartment.

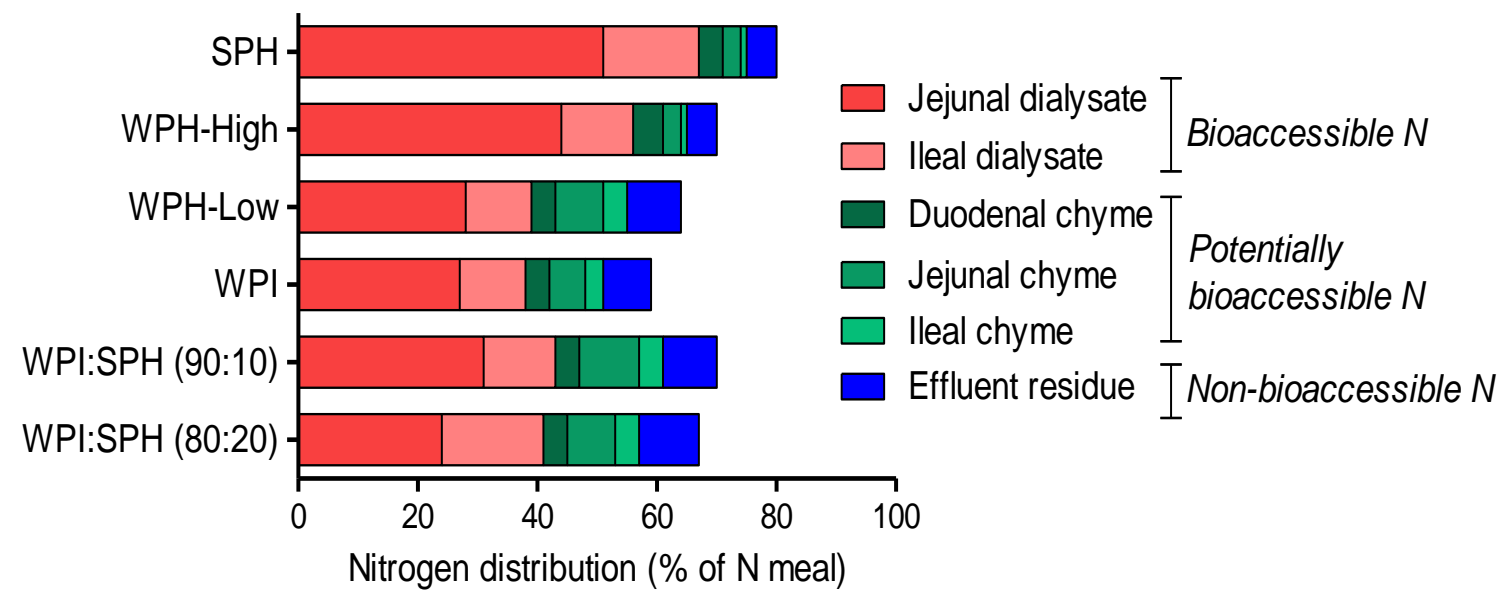

Figure 2. Nitrogen distribution throughout the TIM-1 compartments at the end of the 2 hour digestion. SHP, salmon protein hydrolysate; WPH-High, whey protein hydrolysate extensively hydrolysed; WPH-Low, whey protein hydrolysate weakly hydrolysed; WPI, whey protein isolate.

The amounts of nitrogen remaining in chyme of duodenal, jejunal and ileal compartments reflected those measured in the dialysates since TIM-1 compartments are considered as communicating vessels when the absorption process started in the jejunal region. For SPH and WPH-High, the amounts of nitrogen in chyme (duodenum + jejunum + ileum) were thus lower $(\sim 8.5 \%)$ than in other substrates for which these values varied from $12.7 \%$ to $18.0 \%$. Nitrogen in chyme can be considered as potentially bioaccessible since a variable amount could be transferred in dialysates during a longer digestion. In our study, a fast GI passage (liquid meal) was selected since the main goal to the study was to compare the kinetics of nitrogen absorption from extensively hydrolysed substrates (SPH and WPH-High), which contained high level of low molecular weight peptides. However, if parameters for a solid meal had been selected instead, the transit time would have been longer and would have favoured the passage of nitrogen in chyme through the semipermeable membranes.

The residue flushes out in effluent at the end of the two hour digestion represented the fraction of non-bioaccessible nitrogen in the protein substrates. This fraction is lower in SPH and WPH-High $(\sim 5 \%)$ than in other substrates $(7.9-9.5 \%)$ since higher proportions of nitrogen were found in dialysates and intestinal chyme at the end of the digestion.

Finally, we observed that total nitrogen recovery after the two hour digestion varied from $58.2 \%$ to $79.7 \%$ depending on the protein substrate (Figure 2). These values seemed to reflect the content of bioaccessible nitrogen in the intestinal dialysates, with the lowest value obtained for non-hydrolysed WPI and the highest value for SPH. The amount of non-recovered nitrogen at the end of the experiments could be explained by the amount of nitrogen remaining in the stomach at the end of the experiment (not analysed), but also by the nitrogen losses inherent to the numerous manipulations during the experiments (sampling and analysis). 


\section{CONCLUSION:}

The results of this study clearly indicate that salmon protein hydrolysate provides a higher proportion of bioaccessible nitrogen to a healthy adult compared to non hydrolysed whey protein isolate, but also in comparison with weakly and extensively-hydrolysed whey protein, although these latter sources of proteins are already recognized as being highly digestible.

Specifically, the salmon protein hydrolysate showed higher nitrogen bioavailability compared to whey protein isolate $(+79 \%)$, weakly hydrolysed whey protein $(+74 \%)$ and extensively hydrolysed whey protein $(+20 \%)$. This excellent nitrogen absorbing performance of the SPH can be associated with its high content of very low molecular weight peptides $(50 \%<$ $1000 \mathrm{Da}$ ) which could pass through the semipermeable membrane upon digestion in the TIM-1 system which accurately simulates human digestion.

Our results further showed a $\sim 12 \%$ increase in the percent of nitrogen absorption by the small intestine when $10 \%$ and $20 \%$ of non-hydrolyzed WPI was substituted with SPH. The addition of this SPH actually helped improve the taste of the WPI whereas addition of similar quantities of WPH made the mixtures completely unpalatable. The value for these protein mixtures was also higher (+9\%) than that of commonly used weakly hydrolysed whey protein hydrolysate. Thus, incorporating just $10 \%$ of SPH to WPI increases its nitrogen bioavailability at values higher than weakly hydrolysed whey protein. Increasing the fraction of SPH further may even allow a SPH+WPI mixture to reach the higher value of bioaccessible nitrogen measured for extensively hydrolyzed whey protein, which has very limited uses (typically $<4 \%$ ) in medical foods due to its extremely unpalatable organoleptic profile.

\section{Competing Interests:}

Bomi Framroze is the CSO of Hofseth Biocare where the salmon protein hydrolysate was sourced. The other authors have no financial interests or conflicts of interest.

\section{Authors' Contributions:}

All authors contributed to this study: Bomi Framroze; Patricia Savard; Diane Gagnon; Véronique Richard; Sylvie Gauthier

\section{Abbreviations:}

SPH, salmon protein hydrolysate; WPH, whey protein hydrolysate; WPI, whey protein isolate

\section{REFERENCES:}

1. Minekus M, Marteau P, Havenaar R. A multicompartmental dynamic computer-controlled model simulating the stomach and small intestine. ATLA-Altern Lab Anim 1995; 197-209.

2. Fernández-Garcia E, Carvajal-Lérida I, Pérez-Gálvez A. In vitro bioaccessibility assessment as a prediction tool of nutritional efficiency. Nutr Res 2009; 29: 751-760.

3. Havenaar R. The model selection tool. Dairy Ind Int 1999; 64(6): 33-36.

4. Zeijdner EE, Mohede ICM. Latest tool for screening new clinical foods. A dynamic, computer-controlled model of the human gastrointestinal tract is the most up-to-date technology for testing new foods. New World Health 1999; 105: 1-12.

5. Dickinson PA, Abu Rmaileh R, Ashworth L, Baker RA, Burke WM, Patterson CM, 
Stainforth N, Yasin M. An investigation into the utility of a multi-compartmental, dynamic, system of the upper gastrointestinal tract to support formulation development and establish bioequivalence of poorly soluble drugs. AAPS J 2012; 14: 196-205.

6. Salovaara S, Alminger ML, Eklund-Jonsson C. Prolonged transit time through the stomach and small intestine improves iron dialyzability and uptake in vitro. J Agric Food Chem 2003; 51: 5131-5136.

7. Larsson M, Minekus M, Havenaar R. Estimation of the bioavailability of iron and phosphorus in cereals using a dynamic gastrointestinal model. J Sci Food Agric 1997; 74: 99-106.

8. Verwei M, Arkbage K, Havenaar R, Van den Berg H, Witthöft C, Schaafsma G. Folic acid and 5-methyl-tetrahydrofolate in fortified milk are bioaccessible as determined in a dynamic in vitro gastrointestinal model. J Nutr 2003; 133: 2377-2383.

9. Courraud J, Berger J, Cristol J-P, Avallone S. Stability and bioaccessibility of different forms or carotenoids and vitamin A during in vitro digestion. Food Chem 2013; 136: 871-877.

10. Venema K, Dohnalek MH. Digestibility and availability of nitrogen for whey protein hydrolysate products assessed using a dynamic in vitro gastrointestinal model. J Allergy Clin Immunol 2006; 117: 304.

11. Picariello G, Mamone G, Nitride C, Addeo F, Ferranti P. Protein digestomics: Integrated platforms to study food-protein digestion and derived functional and active peptides. TrAC Trends in Analytical Chemistry 2013; 52: 120-134.

12. Butts CA, Monro JA, Moughan PJ. In vitro determination of dietary protein and amino acid digestibility for humans. Br J Nutr 2012; 108(S2): 282-287.

13. Nabil S, Gauthier SF, Drouin R, Poubelle PE, Pouliot Y. In vitro digestion of proteins and growth factors in a bovine whey protein extract as determined using a computer-controlled dynamic gastrointestinal system (TIM-1). Food Dig 2011; 2: 13-22.

14. Chen L, Hébrard G, Beyssac E, Denis S, Subirade M. In vitro study of the release properties of soy-zein protein microspheres with a dynamic artificial digestive system. J Agric Food Chem 2010; 58: 9861-9867.

15. Blanquet-Diot S, Soufi M, Rambeau M, Rock E, Alric M. Digestive stability of xanthophylls exceeds that of carotenes as studied in a dynamic in vitro gastrointestinal system. 2009; 139 : 876-883.

16. Lila MA, Ribnicky DM, Rojo LE, Rojas-Silva, P, Oren A, Havenaar R, Janle EM, Raskin I, Yousef GG, Grace MH. Complementary approaches to gauge the bioavailability and distribution of ingested berry polyphenolics. 2012; 60: 5763-5771.

17. Blanquet S, Zeijdner E, Beyssac E, Meunier J-P, Denis S, Havenaar R, Alric M. A dynamic artificial gastrointestinal system for studying the behavior of orally administered drug dosage forms under various physiological conditions. Pharm Res 2004; 21: 585-591.

18. Souliman S, Blanquet S, Beyssac E, Cardot J-M. A level A in vitro/in vivo correlation in fasted and fed states using different methods: Applied to solid immediate release oral dosage form. Eur J Pharm Sci 2006; 27: 72-79.

19. Report on Molecular weight and peptide distribution in salmon protein hydrolysate. Green Earth Industries Reports 2008; 1-9.

20. Gauthier SF, Pouliot Y. Functional and biological properties of peptides obtained by 
enzymatic hydrolysis of whey proteins. J Dairy Sci 2003; 86: E78-E87.

21. Liu X, Jiang D, Peterson DG. Identification of bitter peptides in whey protein hydrolysate. J Agric Food Chem 2013; dx.doi.org/10.1021/jf4019728.

22. Leksrisompong P, Gerard P, Lopetcharat K, Drake M. Bitter taste inhibiting agents for whey protein hydrolysate and whey protein hydrolysate beverages. J Food Sci 2012; 77: S282S287.

23. Dumas JBA. Precedes de l'analyse organique. Ann Chim Phys 1831; 47: 198-205. 